COMMENT. Despite the differences in epilepsy terminology, the earlier Lennox and recent Australian twin studies provide similar and valuable evidence of a genetic basis for generalized epilepsies and febrile seizures and further support the concept of specific genes for epilepsy syndromes. A genetic factor in symptomatic generalized epilepsies demonstrated in the twin studies is also apparent in a current Japanese study of family history rates of epilepsy and consanguinity. (Wakamoto H et al. Brain Dev 2004;26:184-189). In 311 probands with childhood-onset epilepsy, a family history of epilepsy (within second-degree relatives) occurred in $19 \%$ and consanguinity (within first-degree relatives) in $6 \%$. A positive family history was more common with generalized than localization-related epilepsies, and more common with idiopathic/cryptogenic epilepsy than symptomatic epilepsy. However, the data suggest that a genetic susceptibility could play a role in the etiology of pre- or perinatal symptomatic generalized epilepsy and in postnatal symptomatic localization-related epilepsy. Also, a genetic factor through consanguinity may influence the etiology of idiopathic/cryptogenic and symptomatic generalized epilepsies.

\title{
SCN2A MUTATIONS AND BENIGN FAMILIAL NEONATAL- INFANTILE SEIZURES
}

SCN2A sodium channel gene was analyzed in 2 families with probable benign familial neonatal-infantile seizures (BFNISs), 9 with possible BFNIS, 10 with benign familial infantile seizures, and in 93 additional families with various early childhood epilepsies, in a study at the University of Melbourne, Australia, and other international centers. Six different SCN2A mutations were found in 8 families and 56 affected individuals with BFNIS, and in no other family in the study. BFNIS is an autosomal dominant disorder that presents from day 2 to 7 months of age (mean, $11.2+/-9.2$ weeks) with afebrile secondarily generalized partial seizures of varying frequency, from a few to clusters of many per day. Seizures completely remit by 12 months of age, with no recurrences in later childhood or adolescence. Interictal EEGs in the active phase were either normal or showed focal epileptiform discharges in posterior or central regions. Brain imaging was normal in 8 cases; one patient had a choroid plexus papilloma. SCN2A mutations are specific for BFNIS. (Berkovic SF, Heron SE, Giordano L, et al. Benign familial neonatal-infantile seizures: Characterization of a new sodium channelopathy. Ann Neurol April 2004;55:550-557). (Respond: Dr Berkovic, Epilepsy Research Centre, Neurosciences Building, Repatriation Campus, Heidelberg West, Victoria 3081, Australia).

COMMENT. Benign familial neonatal-infantile seizure disorder (BFNIS) with onset around 3 months is an intermediate variant of autosomal dominant benign epilepsies in the first year. Benign familial neonatal seizures (BFNS) begin around day 3 and benign familial infantile seizures (BFIS) begin around 6 months. BFNIS has now been linked to mutations in the sodium channel gene SCN2A (Heron et al, 2002; Berkovic 2004), BFNS is caused by defects in potassium channel genes KCNQ2 and KCNQ3 (Singh et al, 1998), and BFIS in one family is associated with a mutation in the ATP1A2 gene (Vanmolkot et al, 2003). BFNIS has characteristic clinical manifestations that allow early diagnosis and an excellent prognosis.

A total of 13 genes have been identified in human idiopathic epilepsies since 1995 (Steinlein OK. Genes and mutations in human idiopathic epilepsy. Brain Dev June 
2004;26:213-218). Most of the known genes code for ion channels, but 2 are not channelopathies (MASS1/VLGR1 coding for a G-protein coupled receptor in one family with febrile and afebrile seizures, and LG11, a tumor suppressor gene, identified in several families with autosomal dominant (AD) familial lateral temporal lobe epilepsy) Other epilepsies with identified genes are AD familial nocturnal frontal lobe epilepsy (CHRNA4), generalized epilepsy with febrile seizures plus (SCN1A, SCN2A), severe myoclonic epilepsy of infancy (SCN1A), and juvenile myoclonic epilepsy (GABRA1). A large number of genetic factors probably contribute to seizure susceptibility.

The clinical spectrum of SCN1A mutations ranges from febrile seizures, febrile seizures plus, a mild and a classical form of severe myoclonic epilepsy in infancy (Dravet syndrome). (Berten PGM et al. Clinical correlations of mutations in the SCN1A gene: from febrile seizures to severe myoclonic epilepsy in infancy. Pediatr Neurol April 2004;30:236243).

\section{EPILEPSY RISK FACTORS FOLLOWING NEONATAL SEIZURES}

Clinical and polysomnographic risk factors as early predictors for the development of postnatal epilepsy were determined in 158 infants presenting with two or more seizures, in a study at Hospital Sao Lucas, Porto Alegre, Brazil. Epilepsy rate after neonatal seizures was $22 \%$ within 12 months follow-up and $33.8 \%$ within 48 months. The types of epilepsy syndromes included: West syndrome in 12 infants; focal symptomatic epilepsy in 10; infantile epileptic encephalopathy in 3; other generalized symptomatic epilepsies in 5; and miscellaneous (13). Perinatal asphyxia, electrolyte imbalance, and bacterial meningitis were the most frequent etiologic factors in neonatal seizures. An abnormal neurologic examination, cerebral palsy, cognitive deficits, and abnormal polysomnographic recording were predictors of an unfavorable outcome and development of postnatal epilepsy. Prematurity, birth weight, and perinatal asphyxia were not significantly related to outcome, but the incidence of postnatal epilepsy was significantly correlated with neonatal bacterial meningitis. Neonates with seizures that required large initial doses and maintenance anticonvulsant drugs were at greater risk of postnatal epilepsy. (Da Silva LFG, Nunes ML, Da Costa JC. Risk factors for developing epilepsy after neonatal seizures. Pediatr Neurol April 2004;30:271-277). (Respond: Dr Nunes, Division of Neurology, Hospital Sao Lucas, Pontificia Universidade Catolica do Rio Grande do Sul School of Medicine, Av Ipiranga 6690, cj220, 90610-000 Porto, Alegre, RS, Brazil).

COMMENT. In this selected population that included infants in intensive care, a higher incidence of epilepsy than that seen in population-based studies could be expected. Multiple risk factors for neonatal seizures, and especially bacterial meningitis, are associated with a higher probability of developing postnatal epilepsy. An abnormal neurologic examination and polysomnographic abnormalities are predictors of an unfavorable outcome.

\section{HYPOTHYROIDISM AND NEONATAL SEIZURES}

A term infant born to a mother with gestational diabetes and a history of hypothyroidism presented with seizures on the $6^{\text {th }}$ day of life that responded to L-thyroxine, in a report from The Children's National Medical Center, Washington, DC. The mother had 